\title{
Geomechanics: from micro to macro editorial
}

\section{IS-Shanghai 2010: International Symposium on Geomechanics and Geotechnics: From Micro to Macro, Shanghai, China, October 2010}

\author{
Mingjing Jiang
}

Published online: 7 September 2010

(C) Springer-Verlag 2010

\section{Conference information}

Organizing Committee:

Prof. Yongsheng Li, Chairman (Tongji University, China)

Prof. Mingjing Jiang, Co-Chairman (Tongji University, China)

Prof. Masayuki Hyodo, Co-Chairman (Yamaguchi University, Japan)

Prof. Hehua Zhu, Co-Chairman (Tongji University, China)

Advisory Committee:

Prof. Malcom Bolton, Chairman (Cambridge University, UK)

Prof. Serge Leroueil, Co-Chairman (Laval University, Canada)

Prof. Zu-yu Chen,Co-Chairman (China Institute of Water Resources and Hydropower Research, China)

Academic Committee:

Prof. Mingjing Jiang, Chairman (Tongji University, China)

Prof. Masayuki Hyodo, Co-Chairman (Yamaguchi University, Japan)

Prof. Stefan Luding, Co-Chairman (Twente University, Netherlands)

Prof. Felix Darve, Co-Chairman (Institut National Polytechnique de Grenoble, France)

M. Jiang (凶)

Department of Geotechnical Engineering, College of Civil

Engineering, Tongji University, 1239 Siping Road,

200092 Shanghai, China

e-mail: mingjing.jiang@tongji.edu.cn

URL: http://geotec.tongji.edu.cn/selfpage/jiangmj/MingjingJiang.htm
Conference website:

http://geotec.tongji.edu.cn/is-shanghai2010/

\section{Theme}

Soils are composed of grains but they are generally treated as continua in the classical framework of geomechanics. Their macroscopic response under loading, such as their non-linearity, yielding and anisotropy, is controlled by their micro-structure, the characteristics of the grains and the disposition of contacts between them. There have been rapid advances in technology both to investigate the microscopic properties of soils, and to simulate their granular behaviour explicitly through Discrete Element Method (DEM). DEM was originally used to reproduce element tests, but it is now being advocated for boundary-value problems. IS-Shanghai 2010 aims to provide an opportunity for the exchange of ideas and information on experiments, numerical models and engineering applications related to the discrete nature of geomaterials.

IS-Shanghai 2010 follows the International Symposium on the Geomechanics and Geotechnics of Particulate Behaviour held at Yamaguchi University in 2006 which was the first to be organized under the auspices of Technical Committee 35 (now designated TC105) of the International Society of Soil Mechanics and Geotechnical Engineering (ISSMGE). The papers in the symposium were organized into three broad themes:

- Micro-/macro-characterization

- Discrete element modeling

- Engineering applications

This special issue deals mostly with Theme 2: Discrete element modeling. The nine papers include DEM results 
in particle shape effects, rolling resistance effects, stressinduced anisotropy, and engineering application of granular flows.

\section{Location}

Shanghai is the largest commercial and financial center in China, situated on the estuary of Yangtze River. It attracts thousands of tourists from all over the world everyday due to its great economic impulse, distinctive attractions as well asits blended culture of the West and the East. In addition, Shanghai is the frontier of applying advanced Geomechanics and Geotechnics in China. Moreover, 2010 is the right time to view several ongoing and exciting projects related to geotechnical engineering there, e.g. World Expo 2010. The Sino-French Center is the international conference hall of Tongji University, situated in the main campus of Tongji University, near to the energetic downtown Shanghai. This beautiful location has helped to create a mostly-relaxed atmosphere that has been ideally suitable for intense discussions and initiation of new collaborations. 